\title{
Significado de ser docente para el adulto mayor sanfernandino
}

\author{
Martha Martina, Gladys Santos, Julia Lema
}

Facultad de Medicina, UNMSM

Objetivos: Comprender el significado de ser docente para el adulto mayor de la Facultad de Medicina de San Marcos y describir las motivaciones que determinan, de alguna forma, su decisión de postergar su retiro, no obstante haber cumplido con los requisitos legales para la jubilación.

Diseño: Estudio de caso cualitativo.

Institución: Facultad de Medicina, UNMSM.

Participantes: Docentes adulto mayores de la Facultad de Medicina.

Intervenciones: Entrevista a profundidad.

Principales medidas de resultados: Se identificó cuatro categorías: exponiendo sus motivaciones para continuar en la docencia siendo adulto mayor; valorando sus vivencias como profesional y como docente; sintiéndose marginado por su edad y sintiendo preocupaciones sociales, políticas, educativas y humanas.

Resultados: Los docentes adultos mayores reconocieron que el ejercicio de la docencia los hacía 'sentir vivos', vitales, en contacto con los jóvenes estudiantes; sentían el 'espíritu universitario', que les permitía continuar siendo productivos para su Universidad y para ellos mismos. Consideraron que la docencia no es transmisión de conocimientos, es educar, es transmitir y compartir valores. Se reconocieron como docentes que sienten la necesidad de compartir con los estudiantes y los jóvenes docentes su amplia experiencia profesional y docente, ser capaces de seguir laborando y con vigencia de sus facultades mentales e intelectuales.

Conclusiones: Los docentes adultos mayores reconocieron ser productivos para la universidad, por la experiencia profesional y docente.

Palabras clave: Adulto mayor, universitarios, jubilación (fuente: DeCS BIREME).

\section{¿Por qué quiero ser obstetra?: motivos de elección de carrera en estudiantes de Obstetricia}

Milena López, Mauro Huamaní, Dora Huapaya, Jenny Zavaleta, Edita Cuya, Carmen Peña, Diana Alegría, Clara Díaz, Luis Ormeño, Marcelina Rosas EAP de Obstetricia, Facultad de Medicina, UNMSM, Lima, Perú

Objetivos: Determinar los motivos por los cuales las estudiantes de Obstetricia elijen su carrera y cómo se ven influenciadas por los estereotipos de género.

Diseño: Transversal.

Institución: EAP de Obstetricia, Facultad de Medicina, UNMSM, Lima, Perú.

Participantes: Estudiantes de Obstetricia.

Intervenciones: Se aplicó un cuestionario auto-desarrollado a 322 estudiantes de Obstetricia, estratificadas proporcionalmente del primer al quinto año de estudio, de tres secciones: 1) Datos sociodemográficos; 2) Inventario de motivos para la elección de una carrera de Salud; y, 3) Inventario de masculinidad y feminidad. Se realizó análisis bivariado con prueba t, chi cuadrado, Kruskal-Wallis con $p<0,05$ y nivel de confianza del $95 \%$.

Principales medidas de resultados: La asociación entre la internalización de estereotipos de género y los motivos de elección de la carrera.

Resultados: La escala de feminidad $(p=0,130)$ y masculinidad $(p=0,036)$ no difirieron entre los estudiantes del primero al quinto año. Los motivos de elección de la carrera fueron: vocación de servicio $(10,29 \pm 1,69)$, interés científico $(9,60 \pm 1,71)$. El rasgo feminidad estuvo asociado a vocación de servicio $(p=0,000)$.

Conclusiones: Las estudiantes de obstetricia presentaron predominancia de rasgos femeninos y eligieron su carrera por vocación de servicio e interés científico.

Palabras clave: Género, estudiantes, obstetricia, IMAFE, elección de carrera, profesiones de la salud. 